\title{
An unexpected complication of a percutaneous coronary angioplasty
}

\author{
Luca Monzo',2, Nino Cocco², Leonardo Calò2 \\ 1 Department of Cardiovascular, Respiratory, Nephrological, Anesthetic and Geriatric Sciences, \\ "Sapienza" University, Rome \\ 2 Department of Cardiology, Policlinico Casilino, Rome, Italy
}

\begin{abstract}
Takotsubo cardiomyopathy (TCM) is characterized by transient ventricular dysfunction, classically in its apical and mid segments in the absence of coronary lesions, and is often observed after intense stressful events and occasionally associated to an acute medical illness. We describe a case of TCM associated with coronary artery disease and triggered by a percutaneous coronary angioplasty. This case highlights the concept that a medical procedure can lead, in certain conditions, to TCM and provides new interesting insights on the pathophysiology of coronary syndromes.
\end{abstract}

\section{Introduction}

De novo ST elevation after a percutaneous coronary angioplasty (PCI) is a very challenging scenario for cardiologists and should be managed carefully as it can be a warning sign of really dangerous PCI complications like stent thrombosis, no reflow, embolization, coronary spasm, coronary or aortic dissection and pericardial effusion.

An increasingly recognized and probably not so rare cause of ST elevation, mostly in postmenopausal woman, is the Takotsubo cardiomyopathy (TCM) [1].

Corresponding author: Dr. Luca Monzo, Department of Cardiovascular, Respiratory, Nephrological, Anesthetic and Geriatric Sciences, "Sapienza" University, Piazzale Aldo Moro 5, 00185 Rome, Italy.

Tel. +39.06.23188406 - Fax: +39.06.23188408.

E-mail: luca.monzo@uniromal.it

Key words: Takotsubo cardiomyopathy; coronary artery disease; percutaneous coronary angioplasty.

Contributions: LM, manuscript writing and data collection; $\mathrm{NC}$, case supervision and data collection; LC, manuscript revision and case supervision.

Conflict of interest: The authors declare no conflict of interest and have not received any funding for this manuscript.

Received for publication: 24 May 2018

Accepted for publication: 31 July 2018

(C) Copyright L. Monzo et al., 2018

Tipografia PI-ME Editrice, Italy

Monaldi Archives for Chest Disease 2018; 88:969

doi: 10.4081/monaldi.2018.969

This article is distributed under the terms of the Creative Commons Attribution Noncommercial License (by-nc 4.0) which permits any noncommercial use, distribution, and reproduction in any medium, provided the original author(s) and source are credited.
TCM was firstly described in Japanese population more than 25 years ago [2] and take its name from the Japanese traditional octopus trap, resembling the shape of the left ventricle during systole on imaging studies due to apical and mid-ventricular dysfunction. Although the exact etiology of TCM is still unknown, the syndrome appears to be triggered by a significant emotional or physical stressor in a context of normal coronary arteries [3], although recently some cases associated with cardiologic invasive procedures have been described $[4,5]$.

\section{Case Report}

A 78-year-old woman with hypertension, typical exertional angina and positive ECG stress test, was admitted to the hospital for elective coronary angiography and possible intervention. Baseline ECG showed normal sinus rhythm and slight ventricular repolarization abnormalities (Figure 1A). Left coronary angiography revealed a 70\% stenosis in the middle part of the left anterior descending coronary artery (LAD) without critical stenosis in the dominant right coronary artery and in the left circumflex coronary artery (Figure $2 \mathrm{~A}$ and video 1 ). Direct sirolimus eluting stenting of the middle segment of the LAD was performed (Figure $2 \mathrm{~B}$ and video 2 ) with excellent results and a final thrombolysis in myocardial infarction (TIMI) 3 flow. The procedure was uneventful.

About 30 min after the angioplasty patient complained heavy headache and constrictive chest pain associated to high blood pressure values (210/100 mmHg). Urgent electrocardiogram (ECG) revealed ST segment elevation in precordial leads (Figure 1B) meanwhile the echocardiogram showed apical and mid wall segments akinesis and basal wall segments hyperkinesis with a typical apical ballooning pattern (video 3 ).

In consideration of the clinical evolution patient was treated with intravenous nitroglycerin and a new angiography was immediately performed. The coronary angiography confirmed the good result of previous intervention with TIMI III flow in the LAD (Figure 3A and video 4) and the ventriculography confirmed a typical apical ballooning pattern (Figure 3B and 3C, video 5). Due to the normal flow in the $\mathrm{LAD}$, it was unlikely to suspect a transient LAD thrombosis, and the extensive and persistent wall motion and ECG abnormality could not be explained on that basis. In consideration of the high value of cardiac magnetic resonance in the differential diagnosis between TCM and acute myocardial infarction we offered the patient to undergo the exam, but she refused because of claustrophobia.

Anyway, the ECG evolution (Figure 1C) and the early partial recovery of the left ventricular kinesis during next days supported the diagnosis of TCM. After few hours from the index event myocardial markers started to rise, with a high sensibility troponin I peak of $276 \mathrm{pg} / \mathrm{mL}$ (cutoff value $<15 \mathrm{pg} / \mathrm{mL}$; ECLIA assay, Roche Diagnostics, UK) the day after and a fast decrease in the following days. The patient's remaining hospital course was uneventful, and she was discharged home in stable conditions on beta blockers, in addition to other necessary medications. Subsequent echocardiogram 1 month later revealed complete recovery of left ventricular systolic function (video 6 ). 


\section{Discussion}

Since its initial description [2] TCM has generated much debate and interest. Over the past two decades, much progress has been made in understanding this syndrome but the etiology is still unclear and there are many variants of this syndrome. Currently, the most commonly ac- cepted theory on the pathophysiology of TCM centers on excess catecholaminergic state and enhanced sympathetic activity [6].

Hyper stimulation of the sympathetic nervous system may also trigger coronary vasospasm, that has been implicated as one of the possible pathophysiological mechanisms in TCM [7,8]. Although it is not possible to definitively exclude that in our report anxiety somatization with massive neurotransmitter discharge caused vasomotricity disturbance,
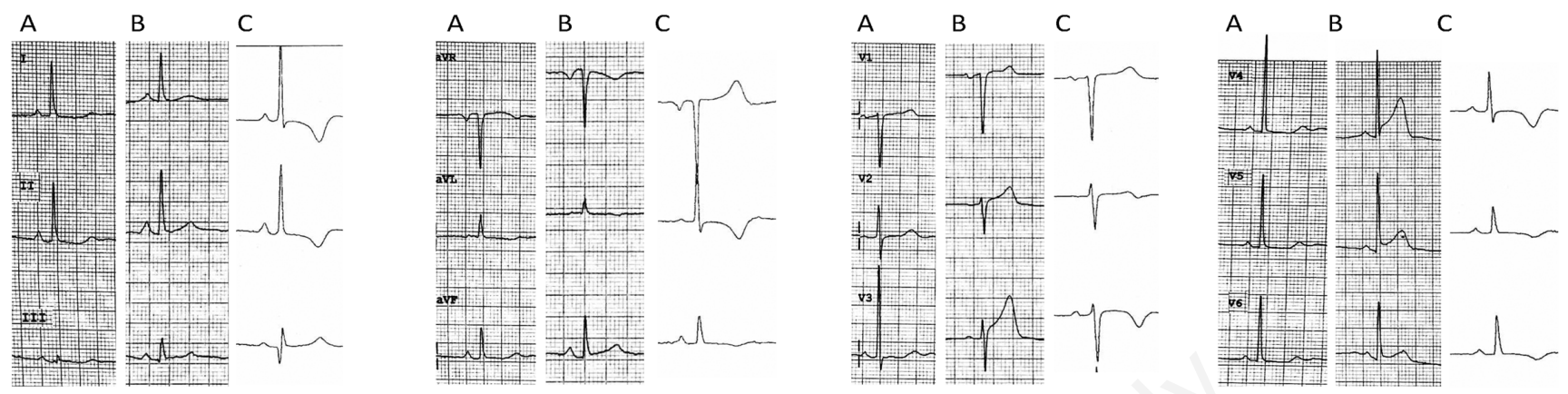

Figure 1. Electrocardiograms evolution. A) Baseline electrocardiogram. B) Urgent electrocardiogram during chest pain episode after PCI- C) Electrocardiogram $24 \mathrm{~h}$ after PCI. PCI, percutaneous coronary intervention.
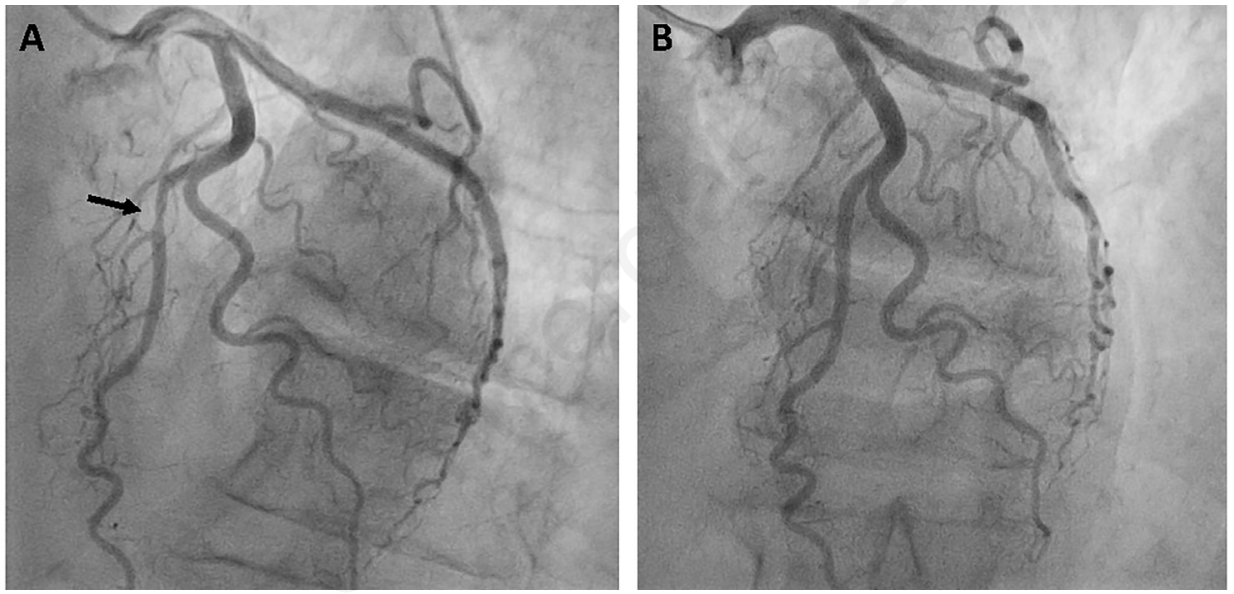

Figure 2. Coronary angiograms pre and post stent implantation. A) The LAD shows critical stenosis in its mid tract (arrow). B) Good result after stent implantation. LAD, left anterior discending artery.
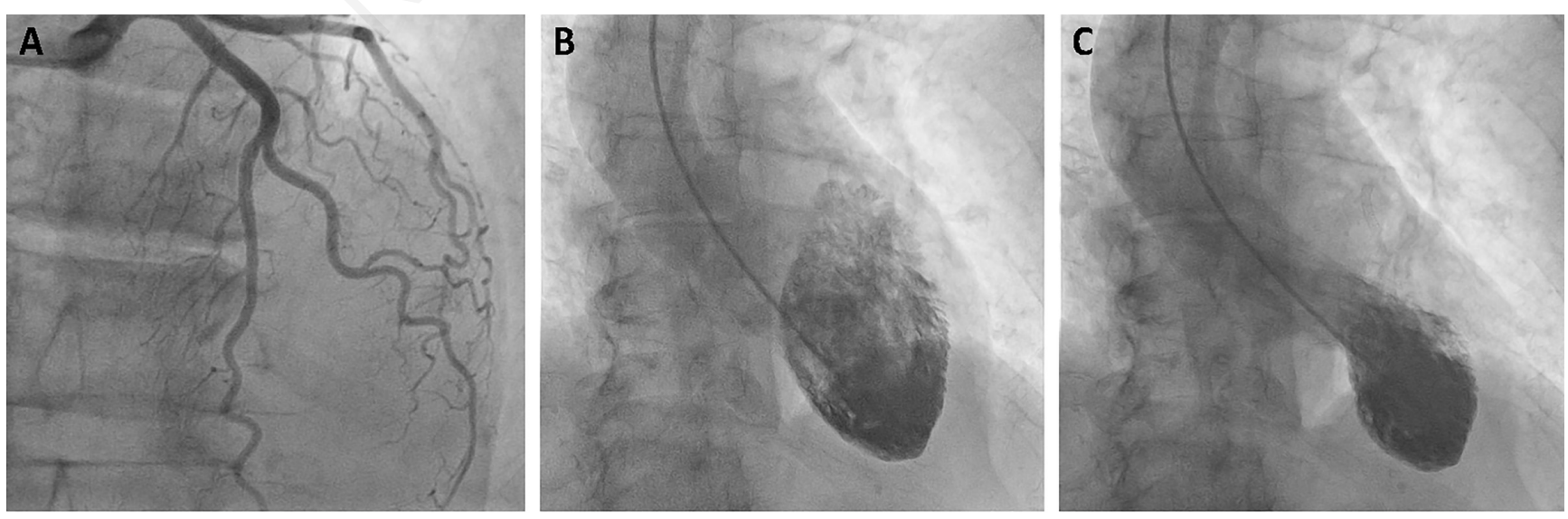

Figure 3. Urgent coronary angiograms show patent stent in the LAD and apical ballooning configuration. A) The LAD shows patent stent and normal flow. B) Left ventricle end-diastolic right anterior oblique view. C) Apical ballooning with hypercontractile basal segments during systole. $\mathrm{LAD}$, left anterior descending artery. 
we maintain it isn't the principal mechanism of this TCM case. In fact, intravenous nitroglycerin didn't modify patient's symptoms or ECG and during urgent angiography there wasn't any evidence of coronary hyper reactivity. Consequently, if any degree of vasospasm occurred, its contribution to the clinical scenario development was negligible compared to other main mechanisms acting directly on cardiomyocytes.

The Mayo criteria for TCM [9] rely on the "absence of significant obstructive coronary artery disease or angiographic evidence of plaque rupture". Since then, there have been multiple case series, challenging the notion that coronary artery disease necessarily excludes the diagnosis of TCM. It is now increasingly accepted that the two conditions are not mutually exclusive but can exist coincidently [10]

We described a typical apical variant of TCM occurred after an uncomplicated coronary angioplasty with direct stenting of LAD ended uneventful and with an optimal angiographic result. Our case should raise attention because remarks the concept that TCM and coronary stenosis are not mutually exclusive, but they can coexist and sometimes be correlated. This idea also applies to acute coronary syndromes, that are usually characterized by a high sympathetic rush. Moreover, in some patients with a short symptom to balloon time and excellent angiographic results extensive electrocardiogram alteration and echocardiographic wall motion abnormality seem not justified by plaque characteristics, thrombus burden, ischemia time or TIMI flow, referring to more sophisticated pathophysiological mechanisms to explain clinical data than the simple coronary artery stenosis.

Obviously, in the acute setting there are very complex and not completely understood alteration of the microcirculatory system and myocardial stunning to consider, but in some cases a certain component of stress cardiomyopathy could explain cases of poor response to a technically successful PCI and provide the basis to new pathophysiological insights, especially in the contest of acute coronary syndromes.

\section{References}

1. Bybee KA, Kara T, Prasad A, et al. Systematic review: transient left ventricular apical ballooning: a syndrome that mimics ST-segment elevation myocardial infarction. Ann Intern Med 2004;141:858-65.

2. Dote K, Sato H, Tateishi H, et al. [Myocardial stunning due to simultaneous multivessel coronary spasms: a review of 5 cases]. J Cardiol 1991;21:203-14.[Article in Japanese].

3. Boland TA, Lee VH, Bleck TP. Stress-induced cardiomyopathy. Crit Care Med 2015;43:686-93.

4. Chiariello GA, Bruno P, Colizzi C, et al. Takotsubo cardiomyopathy following cardiac surgery. J Card Surg 2016;31:89-95.

5. Hussain J, Laufer N, Sorrof S, Pershad A. Takotsubo cardiomyopathy after coronary intervention developed during hospitalization. Ann Thorac Surg 2009;88:e63-5.

6. Wittstein IS, Thiemann DR, Lima JA, et al. Neurohumoral features of myocardial stunning due to sudden emotional stress. N Engl J Med 2005;352:539-48.

7. Haghi D, Suselbeck T, Wolpert C. Severe multivessel coronary vasospasm and left ventricular ballooning syndrome. Circ Cardiovasc Interv 2009;2:268-9.

8. Dores H, Raposo L, Ferreira J, et al. Apical ballooning syndrome during diagnostic coronary angiography. Arq Bras Cardiol 2013;100:e47-50.

9. Scantlebury DC, Prasad A. Diagnosis of Takotsubo cardiomyopathy. Circ J. 2014;78:2129-39.

10. Alfonso CE. Takotsubo cardiomyopathy and coronary artery disease: A meaningful coincidence? J Am Heart Asso. 2016;5 pii: e005131. 